\title{
Time Invariant Scaling in Discrete Fragmentation Processes
}

\author{
B.G.Giraud and R.Peschanski \\ Service Physique Théorique, DSM-CE Saclay, F91191 Gif/Yvette, France
}

Abstract: Linear rate equations are used to describe the cascading decay of an initial heavy cluster into fragments. We consider moments of arbitrary orders of the mass multiplicity spectrum and derive scaling properties pertaining to their time evolution. We suggest that the mass weighted multiplicity is a suitable observable for the discovery of scaling. Numerical tests validate such properties, even for moderate values of the initial mass (nuclei, percolation clusters, etc.). Finite size effects can be simply parametrized.

In this work we consider binary fragmentation processes where any fragment with mass number $k$ breaks into fragments with mass numbers $j$ and $k-j, j=1,2 \ldots k-1$, with a probability $w_{j k}$ per unit of time. It is assumed that $w_{j k}$ is time independent. By definition, $w_{j k}=0$ if $j \geq k$ and $w_{j k}$ is symmetric if $j$ is replaced by $k-j$, naturally. For technical reasons, we will use for $w_{j k}$ the double of the actual transition rate whenever the special case $w_{j, 2 j}$ occurs. Let $N_{j}(t)$ be the multiplicity of fragment $j$ at time $t$ in a process initiated from the decay of a cluster $A$, namely $N_{j}(0)=\delta_{j A}$. The model under study is described by the following set of linear, first order differential equations,

$$
\frac{d N_{j}}{d t}=-c_{j} N_{j}+\sum_{k=j+1}^{A} w_{j k} N_{k}, j=1, \ldots A
$$

with

$$
c_{j}=\sum_{\ell=1}^{j-1} \frac{w_{\ell j}}{2} .
$$

With components $N_{j}, j=1, \ldots A$, for a column vector $|\mathcal{N}\rangle$, the system, Eqs.(1), boils down to $d|\mathcal{N}>/ d t=\mathcal{W}| \mathcal{N}>$ with a triangular matrix $\mathcal{W}$. The general solution of Eqs.(1) is obviously a sum of exponentials whose rates of decay in time are the trivial eigenvalues of the triangular $\mathcal{W}$, namely the diagonal matrix elements $-c_{j}$.

This matrix $\mathcal{W}$ has a remarquable property, namely a fixed left (row-like) eigenstate $\mathcal{M}_{1}$, whose components $\mathcal{M}_{1 j}=j, j=1, \ldots A$, do not depend on the $w_{\ell k}$ 's. This comes from 
the symmetries of $\mathcal{W}$ demanded by the conservation of the total mass $M_{1}=\sum_{j=1}^{A} j N_{j}$, with $d M_{1} / d t=<\mathcal{M}_{1}|\mathcal{W}| \mathcal{N}>$. The corresponding eigenvalue is, naturally, $-c_{1}=0$. It is thus convenient to define the "mass weighted multiplicity" (MWM) vector $U$ with components $U_{i}=i N_{i}$, whose evolution is governed by a matrix $\mathcal{V}$, with matrix elements $\mathcal{V}_{j k}=j \mathcal{W}_{j k} / k$, hence

$$
d U / d t=\mathcal{V} U
$$

The surface under the histogram defined by $U$ is therefore time invariant, $\sum_{i=1}^{A} U_{i} / A=1$. This invariant normalization gives a hint that an analysis of $U$ might be the best way to reveal scaling and even universality properties of the fragmentation process.

For that analysis, we first consider a moment of arbitrary order $q$

$$
M_{q}=\sum_{j=1}^{A} j^{q-1} U_{j}=\sum_{j=1}^{A} j^{q} N_{j}
$$

where the exponent $q$ is any real number. The time derivative of such a moment is

$$
\frac{d M_{q}}{d t}=\sum_{j=1}^{A} j^{q}\left(\sum_{k=j+1}^{A} w_{j k} N_{k}-c_{j} N_{j}\right)=\sum_{k=1}^{A} k^{q-1} U_{k} d(q, k),
$$

with

$$
d(q, k)=\sum_{j=1}^{k-1} w_{j k}\left[\left(\frac{j}{k}\right)^{q}-\frac{1}{2}\right],
$$

where we have used an interchange of indices $j$ and $k$ in the double summation.

The structure of the right-hand-side of Eq.(5) gives a special interest to those cases where $d(q, k)$ shows scaling properties with respect to $k$. For instance ${ }^{1)}$ if a condition $d(q, k) \simeq d(q)$ is met, this approximate independence with respect to $k$ induces an eigenvalue problem where the corresponding (eigen)moments $M_{q}$ are decoupled and decay exponentially with respect to time. A sufficient condition for this is a behavior $w_{j k} \simeq$ $k^{-1} \varphi(j / k)$, whence, when $k$ is large enough,

$$
d(q, k)=\frac{1}{k} \sum_{j=1}^{k-1} \varphi(j / k)\left[\left(\frac{j}{k}\right)^{q}-\frac{1}{2}\right] \simeq \int_{0}^{1} d x \varphi(x)\left(x^{q}-1 / 2\right),
$$


where $x=j / k$. This indicates indeed a limit where $d(q, k)$ becomes $d(q)$, independent of $k$. Accordingly,

$$
\frac{d M_{q}}{d t} \simeq d(q) M_{q}
$$

Such a decoupling of eigenmodes is also found in the QCD theory of energy-momentum fragmentation $^{2}$, where any moments with a (continuous) order $q$ real and positive is an eigenmode, in contrast however with the present discrete representation of fragmentation where only special values of $q$ can be selected ${ }^{1)}$.

In this letter, we study a more general case where $d(q, k) \simeq k^{a} d(q)$ and $a$ is any real number a priori. This occurs in particular if $w_{j k} \simeq k^{a-1} \varphi(j / k)$. In such a case the resulting evolution equation for moments reads

$$
\frac{d M_{q}}{d t}=\sum_{k=1}^{A} k^{q-1} U_{k} d(q, k) \simeq d(q) M_{q+a}
$$

Here $d(q)$ is still taken from the right-hand side of Eq.(7). This property, Eq.(9), occurs in continuous systems ${ }^{3)}$. Our aim is to rather investigate discrete fragmentations as well.

Let us assume temporarily that Eq.(9) is an exact result. This leads to now wellknown ${ }^{3)}$ solutions with scaling properties,

$$
M_{q}(t)=[s(t)]^{q-1} m_{q}, \quad s(t)=\left([s(0)]^{-a}+a \omega t\right)^{-1 / a}, \quad m_{q+a}=m_{q}(1-q) \omega / d(q),
$$

where $s$ represents an average for the cluster sizes at time $t$. The recursion relation between the coefficients $m_{q}$ relates the various moments to one another via the function $d(q)$, see Eqs. $(7,9,10)$, which contains the dynamical information about the system. Finally $\omega$ is an integration constant. Such scaling solutions for continuous systems are valid only if $a$ is a strictly positive number.

In our present study, the physical quantities under observation are the $U_{i}$ 's, governed by Eq.(3). The translation of Eq.(10) in terms of $U_{i}$ clearly reads

$$
U_{i}(t) \propto[s(t)]^{-1} f[i / s(t)]
$$


where $f$ can be deduced from $d(q)$, but needs not be explicit in a numerical verification of scaling. Indeed, if one solves Eq.(3) numerically, it is sufficient to observe that, for different times $t$, the various $U_{i}(t)$ show similar shapes when referred to the new scaling variable $y \equiv i / s(t)$. Naturally, for each time $t$, one needs a knowledge of $s(t)$, by numerical derivation if necessary.

In this letter, we report numerical results for a two-parameter class of models where

$$
w_{j k}=k^{a-1}\left[(j / k)^{-b}+(1-j / k)^{-b}\right] / 2 .
$$

Such an ansatz for $w_{j k}$ was already investigated with interesting results for the possible existence of eigenmoments ${ }^{1)}$. We first calculate Eq.(3) for many values of $A, a, b, t$, then, as a rule of thumb, discard both $U_{1}$ and $U_{A}$. This is because an obvious peak in the $U$-histogram exists at $U_{A}$ for small $t$ 's and at $U_{1}$ for large $t$ 's. We are rather interested in identifying phenomenologically $s(t)$ as the position of the intermediate extremum (if it exists and if it is unique) of $U_{i}(t)$ as a function of $i$. The reason for this procedure is the fact that, as already stated, the $U$-histogram has a constant surface. An intermediate bump, or conversely a dip, between the "source peak" $U_{A}$ and the "sink peak" $U_{1}$ is thus likely.

Indeed, as shown by Fig.1a, where $A=24, a=0.5, b=-1$, and $t=1.2,2.2,3.2$, there is an intermediate bump, which smoothly moves towards lighter fragments when time increases. Simultaneously, the source peak at $A=24$ decays, while the height of the fragment bump grows. The case shown by Fig.1b is rather different. There is a flat dip rather than a maximum, when $A=24, a=0.5, b=1$, and $t=0.28,0.36$. The flip from a source peak at shorter times into a sink peak at larger times, see $t=0.44$, is striking. Finally, for Fig.1c, where $A=24, a=-0.2, b=-1$, and $t=9$, 10, (and $t=11$, for comparison) we observe three maxima, namely a source peak, a sink peak and an intermediate flat maximum. It will be noticed that the time scales which govern these evolutions seem to depend strongly on $a$ and $b$.

We now turn to the search for scaling properties. For those histograms at time $t$ which show an intermediate maximum $U_{\max }(t)$ at some mass $i_{\max }(t)$, see Fig.1a and 1c, 
we set $s(t)=i_{\max }(t)$. Then the mass scale is defined as $y=i / i_{\max }(t)$, and we consider $\log \left[U_{i}(t) / U_{\max }(t)\right]$ as a function of $y$. For those histograms which show an intermediate minimum, we do the same, except that the reference for scale and normalization is taken with respect to the minimum, naturally.

This allows a comparison of histograms at different times: all histograms reduce to 0 when $y=1$. Finally we discard from our analysis those histograms for which $U_{i}$ is monotonically increasing or decreasing between $i=2$ and $i=A-1$. A few results are shown on Figs.2. Fig.2a exhibits a remarkable match of such "renormalized" histograms for $A=24, a=0.5, b=-1$, and $t=0.8,1.4,2.0$. It must be stressed that the data for $t=0.8$ cover only the interval $0.1 \lesssim y \lesssim 1.3$, while those for $t=1.4$ cover only the interval $0.2 \lesssim y \lesssim 3$, and finally those for $t=2$ cover the interval $0.3 \lesssim y \lesssim 4.5$. This is because the position $i_{\text {max }}$ decreases considerably as a function of time. Hence the mutual continuation of the three curves from one another is all the more striking. Another remarkable match is seen on Fig.2b, where $A=24, a=0.5, b=0.5$, and $t=0.4,0.5,0.6,0.7$.

We have verified that this result does not depend on $A$, but only the parameters $a$ and $b$, which must be in a range $a \gtrsim 0$ and $b<1$. Indeed, as shown for instance by Fig.2c, with $A=24, a=-0.2, b=-1$, and $t=8.7,9.0,9.3$, the overlap of the renormalized histograms is less convincing. Indeed, it is seriously violated in the region of small fragments $y \lesssim 0.2$. Finally, the results obtained when there is a minimum rather than a maximum give no evidence for universality of shapes in such cases, and strongly point to the contrary, see Fig.2d with $A=24, a=0.5, b=1$, and $t=0.24,0.28,0.32$.

For comparison and contrast with Fig.2a, we show in Fig.3 the same attempt for mutual continuation with $A=24, a=0.5, b=-1$, but $t=1.8,2.2,2.6$. Some success is obtained for $y<1$, but the attempt fails for $y>1$. It can be concluded that a "universal" shape of the renormalized histograms is likely for intermediate times, but not for longer times.

It is now interesting to confront our numerical findings with the solutions in the continuous limit ${ }^{3)}$ where Eq.(9) is an exact property of the system. 
i) scaling case: $a>0, b<1$

In the continuous limit, one obtains the scaling behavior described by Eqs.(10,11). If we assume for $f$ a simple factorized form and perform the same rescaling transformation as for the distributions $U_{i}$, we get

$$
z(y) \equiv\left(1-b^{\prime}\right) / a^{\prime}\left(1-y^{a^{\prime}}+a^{\prime} \log y\right),
$$

where the parameters $a^{\prime}, b^{\prime}$ are to be compared with those $a, b$ of the continuous limit and $z$ is the scaling function emerging from the time-dependent distributions $\log \left[U_{i}(t) / U_{\max }(t)\right]$. A comparison between $z$ and $\log \left[U_{i}(t) / U_{\max }(t)\right]$, see Fig.(4), shows that the rescaled histogram $\log \left[U_{i}(t) / U_{\max }(t)\right]$ is well reproduced by $z$, Eq.(13), with $b^{\prime}=b$, but $a^{\prime} \simeq$ $0.35<a=0.5$. Indeed, in the continuous limit, the scaling function $z(y), y \equiv i / s(t)$, see Eq.(11), is known to behave as $z \propto(1-b) \log y$ for small $y$ and $z \propto-y^{a}$ for large $y$. It seems that the finite size effects do not affect the scaling properties that we have thus observed in the considered cases, albeit possibly modifying some of the scaling indices.

ii) shattering case: $a<0, b<1$

In this case, scaling is approximately valid in the region $y>1$ and strongly violated in the small $y$ region. As a matter of fact, from fitting Fig.2b, we find empirically that the parametrization, Eq.(13), gives a satisfactory agreement for $a^{\prime} \simeq 0.1$ in the region $y>1$ but fails for $y<1$. Note again that $a^{\prime} \neq a$. In the continuous limit, it is known that a shattering transition ${ }^{4)}$ takes place, whereby a part of the system is quickly transformed into a powder of infinitesimal constituents. In the present discrete case, the behavior of $U$ for small values of $y$ can be traced back to a similar formation of a sector of lightest fragments of unit mass.

iii) non-scaling (evaporation) case: $b>1$

The absence of a scaling behavior is likely due to the presence of a minimum instead of a maximum in the function $z(y)$. It is to be noted that the marginal value $b=1$ corresponds to the field-theoretical case mentionned in reference ${ }^{2}$. However, it is not excluded that a partial scaling could be restored by some different empirical definition of the rescaling 
procedure. Nevertheless, the fact that the values $b>1$ give a strong enhancement to the contributions of the very small (and thus very large by symmetry) fragments at the vertex, see Eq.(7), is a hint towards a difference between the continuous and the discontinuous cases.

\section{Discussion and Conclusion:}

We have discovered that, at those intermediate times where a sufficient amount of intermediary mass fragments has been generated (and evaporation and/or shattering are not dominant), the multiplicity histogram has a stable shape. This shape can be parametrized by simple functions and simple parameters and evolves in time under simple scaling rules. This result has been observed numerically for many initial masses, even moderate ones, and a fairly general class of models for the fragmentation vertices. The result is also true for several excursions out of this class, which cannot be reported here in detail. It is thus reasonable to conjecture that a central limit theorem, due to the semi-group, iterative nature of the binary fragmentation cascade, is at work when the intermediary mass fragments build a bump in the mass weighted multiplicity histogram.

We have found that our phenomenology of discrete fragmentation shows both similarities and differences with the description ${ }^{3)}$ proposed for continuous systems. For example, similarities are found when the initial mass is very large, naturally. Differences are found for moderate initial masses. For instance we find no obvious scaling if the histogram has a minimum (evaporation), and conversely, we find a residual scaling for part of the histogram in the presence of shattering. In those cases where there is an agreement between our description and that of the continuous limit, we find nonetheless finite size corrections in the behavior of the scaling curve.

Besides the conjectured existence of a suitable central limit theorem, this work opens several lines of investigation. One one hand, those multifragmentation data which contain sizable numbers of intermediate mass fragments should likely be analyzed in terms of scaling. A (nuclear, atomic) clock is not necessary for dating the corresponding histograms, since our scheme is actually time independent. On the other hand, one should investigate whether binary ${ }^{5)}$ cascades make a sufficient model, or whether fragmentation 
is very sensitive to many-body effects in the medium ${ }^{6}$ ). Comparison with the properties of percolation $^{7)}$ is also in order.

\section{References}

[1] B.G. Giraud and R. Peschanski, Phys.Lett. B 315 (1993) 452.

[2] G. Altarelli and G. Parisi, Nucl. Phys. 126 (1977) 297. V.N. Gribov and L.N. Lipatov, Sov. Journ. Nucl. Phys.15 (1972) 438 and 675. For a review and references, Basics of perturbative QCD Y.L. Dokshitzer, V.A. Khoze, A.H. Mueller and S.I. Troyan (J. Tran Than Van ed. Editions Frontières, France, 1991.)

[3] Z. Cheng and S. Redner, J. Phys. A: Math. Gen. 23 (1990) 1233.

[4] E.D. Mc Grady and Robert M. Ziff Phys. Rev. Lett. 58 (1987) 892.

[5] On a phenomenological ground in relation to nuclear multifragmentation, the problem has been raised by: J. Richert and P. Wagner, Nucl. Phys. A 517 (1990) 299.

[6] D.H.E. Gross et al., Annalen der Physik 1 (1992) 467.

[7] X. Campi, Phys. Lett. B 208 (1988) 351, and contributions to the the Proceedings of Varenna 1990 and 1992 Summer Courses of the International School of Physics Enrico Fermi. For a general review on percolation: D. Stauffer, Introduction to Percolation Theory (Taylor and Francis, London and Philadelphia, Penn. 1985).

\section{Figure Captions}

\section{Fig.1a}

Mass weighted multiplicity $U_{i}$ as a function of fragment mass $i$ for initial mass $A=24$, and vertex parameters $a=0.5$ and $b=-1$. See Eqs.(3,12). Notice how the "source peak" $U_{24}$ decreases when time $t$ increases from $t=1.2$ to $t=3.2$. Simultaneously, a population of intermediate fragments moves into lighter ones.

\section{Fig.1b}

Same as Fig.1a, but $b=1$. In contrast with Fig.1a, the source peak feeds directly the population of lightest fragments and even disappears for $t=0.44$. The process is reminiscent of evaporation. 


\section{Fig.1c}

Same as Fig.1a, but $a=-0.2$. Both source and sink peaks are present, together with an intermediate, temporary maximum, see $t=9,10$. A shattering phenomenon is likely in this case.

\section{Fig.2a}

Scaling comparison of mass weighted multiplicities at various intermediate times. For each time we plot $\log \left(U_{i} / U_{\max }\right)$ as a function of $y=i / i_{\max }$. The parameters are $A=24, a=$ $0.5, b=-1, t=0.8,1.4,2$. Notice how a unique scaling curve emerges from overlapping segments of curves.

\section{Fig.2b}

Same as Fig.2a, but $b=0.5, t=0.4,0.5,0.6,0.7$. Notice how intermediate and lower mass fragments follow scaling. The population of the highest masses deviates from the scaling curve, but its decay nonetheless contributes to it.

\section{Fig.2c}

Same as Fig.2a, but $a=-0.2$. Scaling is likely preserved for $y \gtrsim 0.8$, and strongly violated for $y \lesssim 0.3$. This indicates shattering.

\section{Fig.2d}

Same as Fig.2a, but $b=1$. Absence of scaling when referred to the minimum of $U_{i}$.

\section{Fig.3}

Same as Fig.2a, but larger time intervals. Evidence of scaling violation when $y>1$.

\section{Fig.4}

Same as Fig.2a, but comparison with the theoretical (dashed line) estimate $z(y)$, see Eq.(13). 